\title{
Tankyrases as modulators of pro-tumoral functions: molecular insights and therapeutic opportunities
}

\author{
Esteban Zamudio-Martinez ${ }^{1,2}$, Ana Belén Herrera-Campos ${ }^{1}$, Alberto Muñoz ${ }^{2,3}$, \\ José Manuel Rodríguez-Vargas ${ }^{1,2^{*}}$ and F. Javier Oliver ${ }^{1,2^{*}}$ (D)
}

\begin{abstract}
Tankyrase 1 (TNKS1) and tankyrase 2 (TNKS2) are two homologous proteins that are gaining increasing importance due to their implication in multiple pathways and diseases such as cancer. TNKS1/2 interact with a large variety of substrates through the ankyrin (ANK) domain, which recognizes a sequence present in all the substrates of tankyrase, called Tankyrase Binding Motif (TBM). One of the main functions of tankyrases is the regulation of protein stability through the process of PARylation-dependent ubiquitination (PARdU). Nonetheless, there are other functions less studied that are also essential in order to understand the role of tankyrases in many pathways. In this review, we concentrate in different tankyrase substrates and we analyze in depth the biological consequences derived of their interaction with TNKS1/2. We also examine the concept of both canonical and non-canonical TBMs and finally, we focus on the information about the role of TNKS1/2 in different tumor context, along with the benefits and limitations of the current TNKS inhibitors targeting the catalytic PARP domain and the novel strategies to develop inhibitors against the ankyrin domain. Available data indicates the need for further deepening in the knowledge of tankyrases to elucidate and improve the current view of the role of these PARP family members and get inhibitors with a better therapeutic and safety profile.
\end{abstract}

Keywords: TNKS1/2, Tankyrase binding motif, Proteasomal degradation, Scaffolding function, Cancer, Inhibitors

\section{Background}

Tankyrase 1 (TNKS1) and tankyrase 2 (TNKS2) are two homologous proteins that belong to the Poly (ADP-ribose) polymerases (PARPs) family (also renamed ARTDs), a group of 17 members that catalyze the addition of ADP-ribose molecules into a large and variety amount of proteins, causing a reversible posttranslational modification (PTM) commonly named ADPribosylation $[1,2]$. TNKS1 and TNKS2 share an $81 \%$ nucleotide homology and 85\% amino acid identity [3]. TNKS is located on chromosome 8, contains 1327 amino

\footnotetext{
* Correspondence: jmrodriguez@ipb.csic.es; joliver@ipb.csic.es

${ }^{1}$ Instituto de Parasitología y Biomedicina López Neyra, CSIC, CIBERONC, 18016 Granada, Spain

Full list of author information is available at the end of the article
}

acids and its primary structure can be classified into four different domains: The C-terminal catalytic PARP domain that mediates the PAR addition to its substrates, an sterile alpha module (SAM) responsible for the homo- and hetero-oligomer formation, the ankyrin (ANK) domain divided in 5 clusters (ARC 1-5) which serve as the substrate binding site, and the His, Pro and Ser (HPS) rich domain with unknown function at the Nterminus $[4,5]$. The sequence of HPS domain presents a low complexity, suggesting that it is intrinsically disordered [6]. TNKS2 is situated on chromosome 10, contains 1166 amino acids and presents a similar organization to TNKS. Both catalytic domains are highly conserved, with $94 \%$ homology; the SAM domain has $74 \%$ similarity and the ANK domain shares $83 \%$ identity. 
However, TNKS2 present a different $\mathrm{N}$-terminal region, lacking the HPS domain [5] (Fig. 1a). To date, the FDA (Food and Drug Administration) has approved the use of four PARP inhibitors as a treatment for different tumors: olaparib, rucaparib, niraparib and talazoparib, which have shown a high effectiveness and reduced adverse effects in cases of breast, ovarian and prostate tumors with BRCA1/2 mutations [7, 8]. New groups have focused their projects on the biochemical and functional study of other less characterized members of PARP family, whose clinical potential is in full expansion. Of all of them, the Tankyrase group has a high therapeutic potential in various human diseases. The present review discusses Tankyrases function and the current state of development of chemical inhibitors and their potential as therapeutic targets in cancer.

\section{Tankyrases: the diversity of biological functions and substrates}

TNKS1 has been the best studied of the two isoforms due to its greater abundance. Nevertheless, since their discover, tankyrases have been found to be involved in several functions [9] such as telomere elongation [10], Wnt/ $\beta$-catenin signaling [11], mitosis [12], glucose metabolism [13], pexophagy [14] and DNA repair [15]. Tankyrases interact with all their substrate proteins through the ANK domain, which contains 24 ankyrin repeats that are organized into 5 subdomains, commonly called ankyrin repeat clusters (ARC1-5) [16-18]. It has been described that each ARC works as the basic unit for the recognition of peptides present in tankyrase substrates $[19,20]$. ARCs properties have been studied using peptides and it has been shown that ARC1, ARC2, ARC4 and ARC5 are able to interact with tankyrase partners with a similar binding mode. However, ARC3 maintains a poor conserved binding surface and therefore, it does not bind tankyrase substrates [6, 21]. In fact, more studies are needed to elucidate the ARC3 function, since it might help the rest of ARCs to the full-length protein binding. TNKS1 and TNKS2 seem to have most binding partners in common and a similar localization, including Golgi complex [22], cytoplasm [23], peroxisomes [14], telomeres [24], centrosomes and nuclear pores [25]. Therefore, TNKS1 and TNKS2 may share most of their functions and one tankyrase could substitute for one another, although it has also been shown that both tankyrases are required to maintain some specific functions [23]. An amino acid sequence present in most tankyrase substrates has been identified. This sequence is called Tankyrase Binding Motif or TBM and Guettler and colleagues defined the consensus TBM as a sequence of eight residues RXXPDGXX. However, another kind of TBM different to the consensus or canonical TBM has been described. These non-canonical TBMs contain additional amino acids between the key arginine and glycine residues, but they still behave and bind to the ANK domain of TNKS1/2 in a similar way to the canonical TBM. This information adds new possibilities to the concept of TBM and increases the number of possible tankyrase substrates. Some of them like Axin1 or RNF146 present both kinds of TBMs (canonical and non-canonical). Nevertheless, other proteins like

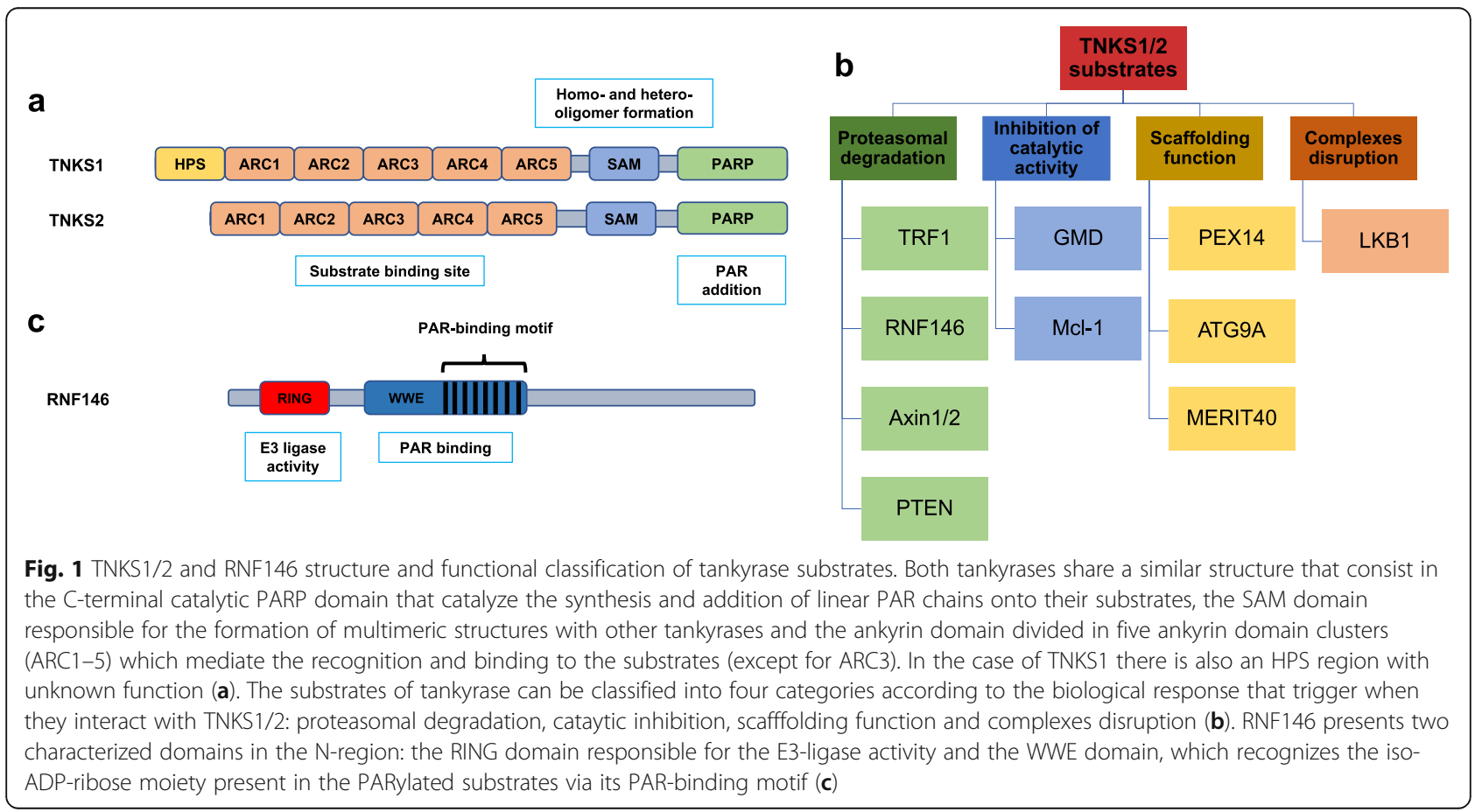


NELFE and IF4A1 only contain non-canonical TBM to interact with TNKS1/2 and we were not considering them as a target $[6,26]$. The TBM also seems to bethe same recognition signal by the four active ARCs [17, 27]. As a member of PARP family, tankyrases can mediate the addition of linear poly (ADP-ribose) (PAR) chains onto their substrates. The linear chains of ADP-ribose are negatively charged and this effect can alter the properties of tankyrase target acceptors [28]. In comparison to the rest of ARTDs, tankyrases do not contain the $\alpha$ helical regulatory domain that controls the catalytic activity. However, several studies have elucidated the role of tankyrase domains in order to regulate its catalytic activity. The SAM domain allows the formation of large molecular TNKS1/2 complexes, which enhance the PARP activity of tankyrase $[29,30]$. TNKS1 can dissociate these complexes through autoPARylation, thus, regulating its own catalytic activity. The ANK domain also helps selecting TNKS1/2 binding partners to be PARylated. This fact is supported by the idea that each active ARC has micromolar affinity for tankyrase substrates and the spatial position of each ARCs is essential for the binding mode of tankyrase with its targets. A model based on crystallography and small-angle-X-ray scattering (SAXS) showed that ARC1, ARC2 and ARC3 present a rigid conformation, with a broken helix between ARC1 and ARC2 and a continuous helix between ARC2 and ARC3. Conversely, ARC4 and ARC5 present a more flexible conformation in comparison to the ARC1-3 module. ARC2 and ARC4 present high affinity for binding target peptides, while ARC1 and ARC5 show low affinity. All this information point out the ANK domain of tankyrase as a multivalent binding system with an special structure that explains how all ARCs collaborate in order to interact with the target partners and also the position of tankyrases to PARylate their substrates. The information about the specific interaction between Axin1 and TNKS1 gave the idea that the binding mode of Axin1 promotes its PARylation because its optimal orientation brings the catalytic domain closer to the target protein. The presence of one or more TBM and their spatial positioning could modulate the interaction with TNKS1 and enhance its catalytic activity $[6,31]$ The recognition of the TBM by TNKS1/2 triggers diverse biological responses including proteasomal degradation, tankyrase catalytic inactivation, scaffolding function or complexes disruption [17] (Fig. 1b and Table 1).

\section{Proteasomal degradation}

One of the best characterized functions of tankyrases is the regulation of protein stability via proteasomal degradation. PARylation by tankyrase is tightly linked to ubiquitination by an E3 ubiquitin ligase called RNF146 (RING finger protein 146) or Iduna. Tankyrases recognize their binding partners and PARylate them through their ANK domain and PARP domain, respectively. Then, RNF146 recognizes PARylated proteins by its WWE domain, which specifically recognizes the isoADP-ribose moiety present only in PAR chains (Fig. 1c). RNF146 catalyzes the formation of a poly-ubiquitin chain linked by its Lys48 residues, in order to trigger the degradation of the substrate by the $26 \mathrm{~S}$ proteasome. This process is commonly called PAR-dependent ubiquitination or PARdU and was discovered through the

Table 1 Tankyrase binding motifs of tankyrase substrates

\begin{tabular}{lllll}
\hline Substrate & PARylated & Biological response & TBM & References \\
\hline TRF1 & Yes & Proteasomal degradation & RGCADG & [27, 32] \\
3BP2 & Yes & Proteasomal degradation & RSPPDG & [17] \\
RNF146 & Yes & Proteasomal degradation & TBM1: RESSADG & TBM3: RSHRGEG \\
& & & TBM4: RSVAGG & TBM5: RSRRPDG \\
& & & TBM1: RPPVPG \\
Axin1 & Yes & Proteasomal degradation & TBM2: RRSDLDLGYEPEG \\
PTEN & Yes & Proteasomal degradation & RYQEDG \\
Mcl-1 & No & Inhibition of TNKS1/2 catalytic activity & RPPPIG \\
GMD & No & Inhibition of TNKS1/2 catalytic activity & RGSGDG \\
PEX14 & No & Scaffolding function & TBM1: RMEVQG \\
ATG9 & No & TBM2: RRGGDG \\
MERIT40 & Yes & Scaffolding function & RLPGLG \\
LKB1 & Yes & Scaffolding function & TBM1: RSNPEG \\
& & TBM2: RSEGEG & [35] \\
\hline
\end{tabular}


implication of RNF146 and TNKS1/2 in the degradation of Axin1 [40-42]. Several groups have shown that a chain of at least four Lys48-linked ubiquitins is necessary to achieve proteasomal degradation [21]. Up to now, it has been described that the stability of many proteins are regulated via PARdU (Table 1), including Axin1/2 [40], PTEN [34], 3BP2 [17], TRF1 [24] or even TNKS1/2 and RNF146, since both are substrates of one another [41, 43] (Fig. 2a).

TRF1 The telomeric repeat-binding factor 1 or TRF1 is a member of the six-subunit shelterin complex responsible for the protection and replication of telomeres. This factor contains a TBM indispensable for the recognition by tankyrase $[27,32]$ (Table 1). A crystal structure of TRF1-TNKS1(ARC2-3) complex revealed additional interactions between the ANK domain of TNKS1 and flanking residues of the TBM present in TRF1, indicating not only the importance of TBM, but surrounding residues to the TNKS1-TRF1 interaction [44]. The role of TRF1 and tankyrase has been related to different functions. Firstly, tankyrase was discovered as a positive regulator of telomerase due to its implication in the releasing from telomeres and degradation of TRF1 [5].
TRF1 is considered a telomerase inhibitor. It has been described that the loss of TRF1 by TNKS1/2 leads to the telomerase upregulation and thus, telomeres elongation [28]. Tankyrase 1 has also been involved in resolving sister telomeres at mitosis because TRF1 and TIN2, another member of shelterin complex, are associated to SA1, one subunit of the cohesin complex. Cohesins are the proteins that maintain sister chromatids together from their replication in $\mathrm{S}$ phase until mitosis, when they are segregated to daughter cells. TNKS1 is responsible for the TRF1-TIN2-SA1 dissociation via TRF1 PARylation, allowing sister telomeres separation [12, 45]. More recently, TNKS1 has been implicated in the DNA Damage Response (DDR) mechanism at telomeres. Specifically, when an oxidative DNA damage causes a Single Strand Break (SSB) in telomeres, TNKS1 is recruited to the area via TRF1 interaction. Then, TNKS1 PARylates TRF1 and the linear poly (ADP-ribose) chain acts as a primary signal to recruit proteins implicated in Single Strand Break Repair (SSBR) like XRCC1 and Pol- $\beta$. Despite PARP1 has been considered the key master of DDR mechanism, the inhibition or silencing of PARP1 does not alter the recruitment of XRCC1 to the damaged telomeres [15]. This research suggests a new mechanism

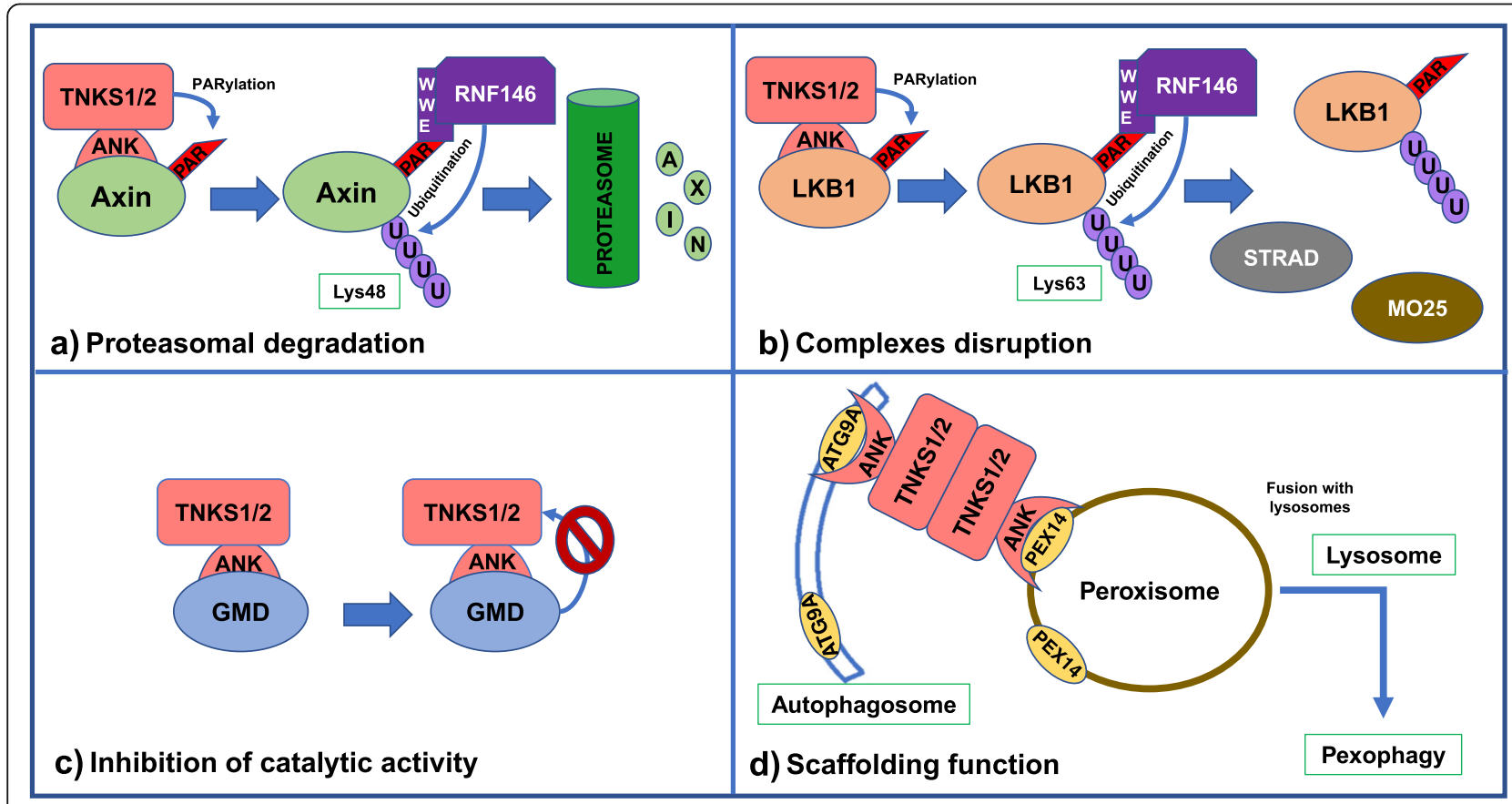

Fig. 2 Biological responses triggered after the recognition of TNKS1/2 substrates. Tankyrases recognize their binding partners via the ANK domain. The most studied function of TNKS1/2 is the regulation of protein stability via proteasomal degradation. Tankyrases PARylate many substrates like Axin1/2, PTEN or 3BP2 and then, the E3 ubiquitin ligase RNF146 recognizes the PARylated proteins and adds a poly-ubiquitin chain linked by its Lys48 residues, which trigger their proteasomal degratation (a). More recently, LKB1 was shown to interact with TNKS1/2 and RNF146, but in this case, tankyrase is able to PARylate LKB1 and RNF146 adds a poly-ubiquitin chain linked by Lys63 in orde to disrupt the complex formed by LKB1, STRAD and MO25 (b). The interaction between tankyrase and the proteins GMD and Mcl-1 causes the inhibition of the catalytic activity of TNKS1/2 (c). Tankyrases also can work as scaffolding proteins via their ANK domain, promoting the interaction between different proteins like PEX14 and ATG9 (without PARylation) or MERIT40 (low levels of PAR) (d) 
for TNKS1 in the initial phase of SSBR at telomeric sites, which contributes to genome stability, cell survival and is independent of cell cycle and telomerase.

RNF146 RING finger protein 146 (RNF146) or Iduna is a RING E3 ubiquitin ligase responsible for the protein ubiquitination. This posttranslational modification is a tight regulated process and is implicated in a large variety of cellular functions carried out by a complex of proteins. It is necessary an E1 ubiquitin activating enzyme, an E2 ubiquitin conjugating enzyme and an E3 ubiquitin ligase, which confers the substrate specificity [46]. RNF146 structure can be divided in two parts: the $\mathrm{N}$-terminal region and the $\mathrm{C}$-terminal region. The $\mathrm{N}$ terminal region contains two characterized domains, the WWE domain and the RING domain, which control the PAR binding function and the E3 ligase activity, respectively (Fig. 1c). The C-terminal domain seems to be intrinsically disordered, which consist in a lack of a fixed three dimensional structure [33, 47]. The WWE domain is also present in some PARP family members, although is less studied. For example, the WWE domain of PARP11 has been described to interact with the ADPr subunit, while the WWE domain of RNF146 recognizes the iso-ADP-ribose moiety, the shortest internal unit present only in PAR chains [42, 48, 49]. RNF146 function is closely related to the PARylation by Tankyrases through the process of PARdU $[40,41]$. Once the target protein is PARylated, RNF146 recognizes and binds to iso-ADPr unit via its WWWE domain. It has been shown that the iso-ADPr moiety also interacts with the RING domain. The ligand binding leads to a conformational change that activates RING domain and this modification results in the allosteric switch that increases the E3 ligase activity of RNF146. This mechanism explains how both WWE and RING regions collaborate to regulate the catalytic activity of RNF146 [33]. Moreover, RNF146 is another substrate of tankyrase. Five possible TBM were observed in the disordered $\mathrm{C}$-terminal region of RNF146 (TBM 1-5), although the TBM4 is the only canonical TBM, while the rest of candidates (TBM 1-3 and 5) are one or two residues longer than the canonical TBM (Table 1). According to a recent study, TBM 1 and 4 seem to be the most important for the TNKS1RNF146 interaction, but the largest effect on TNKS1 binding was found when TBM1 was mutated and not TBM4, which is a consensus TBM. TBM 3 and 5 also present additional contributions to the interaction between tankyrase and RNF146. Nevertheless, TBM2 does not appear to contribute to the interaction with TNKS. This study revealed a new TBM model that presents one extra residue in the middle of the canonical TBM sequence. The RNF146 (TBM1) - TNKS1 (ARC2-3) interaction model showed that extended TBM binds to
ARC2 with an affinity comparable to consensus TBMs. Furthermore, the RNF146-TNKS1 (ARC1-5) interaction model supports the idea of RNF146 using multiple motifs to binding multiple ARCs of tankyrase simultaneously [26].

Axin1/2 Axis inhibition 1 and 2 (Axin1/2) are considered negative regulators of the canonical Wnt pathway $[50,51]$. $\beta$-catenin is the key master of Wnt signaling and is tightly regulated by the $\beta$-catenin destruction complex, which is composed by APC, CK $1 \alpha$, GSK $3 \beta$ and Axin, being the last one the rate-limiting factor. This complex mediates the phosphorylation of $\beta$-catenin, which then is recognized and ubiquitinated by the ubiquitin ligase $\mathrm{SCF}^{\beta-\operatorname{TrCP}}$ in order to target it for proteasomal degradation [52]. Axin was the first well-defined target of PARdU, since TNKS1/2 and RNF146 mediate its degradation via proteasome [11, 40, 41, 47]. For this reason, TNKS1/2 are considered positive regulators of the canonical Wnt pathway. Two TBMs have been described into the Axin $1 / 2$ amino acid sequence. One of them is a canonical TBM, while the other one is a noncanonical TBM, with four additional residues between the key Arg and Gly residues (Table 1). These TBM are conserved in the Axin family and between species. Several crystal structures analysis combined with mutational assays between TNKS1 and Axin1 have demonstrated that both TBM are necessary to mediate a bivalent interaction with the ANK domain of tankyrase and regulate Axin turnover [6, 50].

PTEN Phosphatidylinositol (3,4,5)-trisphosphate phosphatase or PTEN is an enzyme that acts as a negative regulator of the PI3K-Akt signaling pathway [53]. PTEN mediates the dephosphorylation of phosphoinositides to antagonize the kinase PI3K [54]. This pathway controls a variety of processes related to cell cycle and cellular proliferation, thus PTEN is considered a tumor suppressor [55]. PTEN activity is regulated by several posttranslational modifications such as acetylation, ubiquitination, SUMOylation or phosphorylation [56-58]. These modifications are able to control the stability of the enzyme, since some studies revealed the implication of phosphorylation in the regulation of the ubiquitin-mediated degradation $[53,59]$. Recently a new modification of PTEN, TNKS1/2-dependent PARylation, has been discovered. PTEN contains a TBM at the $\mathrm{N}$-terminal region, close to the phosphatase domain (Table 1). It has also been shown the implication of RNF146 in the recognition of PARylated PTEN and its ubiquitination and degradation via proteasome. An in vitro ribosylation assay of PTEN by both TNKS1/2 detected three different PAR acceptor sites by tankyrases (Glu40, Glu150 and Asp326). Furthermore, in vivo ubiquitination assays with mutations 
in different lysine residues of PTEN showed that the major sites for PTEN ubiquitination mediated by RNF146 are Lys342, Lys344 and Lys349 [34].

\section{Other biological responses triggered after the interaction with tankyrases}

Despite our knowledge about tankyrases is increasing, some aspects remain unclear. One of them is how regulatory domains control TNKS1/2 catalytic activity. The ANK domain of TNKS1/2 is responsible for the interactions with a large variety of functional and structural binding partners [6, 32]. Most TNKS1/2 substrates have been shown to be PARylated. Moreover, the most characterized biological response triggered after PARylation is the recognition by the E3 ubiquitin ligase RNF146, which triggers the Lys48-linked ubiquitination and the proteasomal degradation of the target [43]. However, several evidences suggest this is not the only biological response. A recent study revealed LKB1 as a new tankyrase binding partner that after being PARylated, it was recognized by RNF146, but it was not degraded [39]. Other proteins like Mcl-1 [35], PEX14, ATG9A [14], MERIT40 [37] and the enzyme GDP-Mannose-4,6Dehydratase [36] are also able to engage TNKS1/2 without being modified, or even can inhibit the catalytic activity of tankyrase, indicating that different parameters determine TNKS1/2 interaction and/or PARylation (Fig. $1 b)$.

\section{Complexes disruption}

Liver kinase B1 or LKB1 is a serine/threonine kinase implicated in cellular energy homeostasis and is also the major upstream activator of AMPK [60]. LKB1 regulates the activation of AMPK through its phosphorylation at Thr172 [61]. Activated p-AMPK is able to phosphorylate downstream targets implicated in glycolysis and protein and fatty acid synthesis [62-65], causing a decrease in cell proliferation under normal conditions, although increases cell survival during energy stress conditions [66]. LKB1 presents a weak catalytic activity in vitro and in vivo, and its activation is promoted by the formation of a complex with the proteins STRAD and MO25 [67]. The binding of STRAD to the kinase domain of LKB1 promotes its kinase activity [68] and MO25 increases the effect of STRAD on LKB1 [69]. Phosphorylation of LKB1 at several residues has been associated to different functions. For instance, the phosphorylation at Ser428 is related to the AMPK activation [70]. Recently, LKB1 was shown to be a new tankyrase partner since the inhibition or the double loss of TNKS1/2 but not PARP1/2 inhibition enhances the phosphorylation of LKB1 at Ser428, the phosphorylation of AMPK and the expression of their target genes. Two possible tankyrase-binding motifs were found in the amino acid sequence of LKB1
(Table 1). Both TBMs are highly conserved and are indispensable for the interaction with TNKS1/2. In vitro ribosylation assays along with mass spectrometry and point mutations assays determined that Glu130 and Glu138 are the residues critical for LKB1 PARylation. RNF146 also works together with TNKS1/2 to regulate AMPK activation recognizing PARylated LKB1. However, the loss of RNF146 enhances the phosphorylation of LKB1 at Ser428, the activation of AMPK, but does not alter LKB1 protein levels. An in vivo ubiquitination assay proved that in the case of LKB1, RNF146 does not catalyze the Lys48-linked ubiquitination, which usually triggers proteasomal degradation. Instead, RNF146 regulates AMPK activation via non-degradative mechanism that involves Lys63-linked ubiquitination of LKB1. Crystallography analysis of LKB1 revealed that Glu130 and Glu138 residues are situated near the binding site of LKB1 with STRAD and close to the ATP binding groove of LKB1, respectively. All this information indicates that PARylation of theses residues and subsequent Lys63linked ubiquitination prevent the formation of the LKB1/STRAD/MO25 complex and suppress the kinase activity of LKB1 (Fig. 2b). Therefore, TNKS1/2 and RNF146 modulate the catalytic activity of LKB1 and AMPK activation through the regulation of both complex formation and phosphorylation of LKB1, implicating a new mechanism of PARdU [39].

\section{Inhibition of TNKS1/2 catalytic function}

GDP-Mannose-4, 6-Dehydratase or GMD is an enzyme necessary for de novo synthesis of fucose, a monosaccharide present in several glycoproteins and glycolipids and also the major donor in all fucosylation reactions $[71,72]$. GDP-fucose synthesis is carried out by GMD in the cytoplasm and transported to the endoplasmic reticulum or Golgi apparatus in order to be transferred to acceptor targets related to several biological functions such as leukocyte trafficking, neuron morphology and development [73]. GMD was identified as a tankyrase 1 partner, providing the existence of the association between both proteins during interphase. The analysis of GMD sequence revealed a highly conserved TBM present at the $\mathrm{N}$-terminus (Table 1), which has shown to be essential for interaction with TNKS. Nevertheless, in vitro PARP assays and in vivo analyses probed that GMD is unable to be PARylated by tankyrase. In fact, the binding of GMD to TNKS suppresses its catalytic PARP activity. GMD depletion increases TNKS degradation via proteasome, indicating the influence of GMD in tankyrase autoPARylation and tankyrase-mediate PARylation of its targets. This inhibition is also specific for tankyrase and not for other PARPs like PARP1, since it requires the presence of the TBM in GMD [32, 36]. This enzyme represents a type of substrate that is not 
PARylated after binding to TNKS and besides inhibits its PARP catalytic activity (Fig. 2c). Indeed, this is not the only one described because a previous study detected another protein with a similar behavior. Mcl-1 (myeloid cell leukemia-1) is a member of Bcl-2 family implicated in apoptosis regulation [74]. The full-length protein (Mcl-1 L) has been identified as an antiapoptotic factor, while its short splicing variant (Mcl-1S) presents proapoptotic functions [75]. Both isoforms are substrates of TNKS and contain an identified TBM (Table 1), although they are not PARylation acceptors. Mcl-1 is able to inhibit the catalytic activity of TNKS, but to a lesser extent than GMD. Unlike GMD, it is not clear if the ability of Mcl-1 inhibition depends on its TBM [35]. Whether both partners share the same mechanism of inhibition or is mediated by different factors has to be determined.

\section{Scaffolding function}

The role of tankyrases in the cellular homeostasis is not only defined by the catalytic ability to PARylate a great number of proteins. TNKS1/2 can form multimeric structures with other TNKS1/2 via the SAM domain, acting as scaffolding proteins which promote the interaction between different substrates of tankyrases through the binding of their multiple targets to the ankyrin domain. The first evidence of this new role was the observation that tankyrases also participates in Wnt/ $\beta$-catenin pathway independently of the PARP activity $[31,76,77]$. Furthermore, a proteomic assay of protein complexes associated to TNKS1/2 in both normal and under catalytic inhibition, revealed the interaction with two proteins implicated in pexophagy, PEX14 (Peroxin14) and ATG9A (Autophagy-related protein 9A) [7880]. PEX14 is a peroxisomal membrane protein that forms with another protein called PEX5 a heterodimer implicated in cytosolic material docking and delivery into the lumen of peroxisomes [81, 82]. ATG9A is the only transmembrane protein described among the ATG family and it is required for the formation of autophagosomal structure $[83,84]$. The analysis of the amino acid sequence of PEX14 revealed four potential TBMs, but only two of them are responsible for binding PEX14 to both tankyrases (Table 1), suggesting that these TBMs cooperate to mediate the interaction with TNKS1/2. Unlike PEX14, only one of the three possible TBMs discovered in the sequence of ATG9A is indispensable for its association with TNKS1/2 (Table 1). In addition, an essay with a PAR-inactive mutant of tankyrase showed that its enzyme activity is not necessary for the interaction with both pexophagy-related proteins. According to all the results obtained, TNKS1/2 work as scaffolding proteins that mediate the interaction between the peroxisomal protein PEX14 and the autophagy-related protein ATG9A (Fig. 2d). The formation of ATG9ATNKS1/2-PEX14 complex induces pexophagy in a tankyrase dependent manner, but not depending on its PARP activity, since TNKS1/2 do not PARylate either of two proteins. The E3 ubiquitin ligase RF146, which is tightly linked to TNKS1/2 through the PARdU mechanism, is neither involved in this process of pexophagy. Therefore, the new mechanism described is a noncanonical and a ubiquitination-independent process for pexophagy [14]. More recently, MERIT40 (Mediator of Rap80 Interactions and Targeting $40 \mathrm{kD}$ ) has also been reported to be directly associated with tankyrase. Two possible TBM were found in the amino acid sequence (Table 1), although it was shown that TBM1 mainly mediates the interaction with TNKS1/2. The PAR level detected in MERIT40 was weaker compared to those of other tankyrase substrates and the overexpression of tankyrase did not cause the downregulation of MERI T40. In fact, it has been observed that the complexes formed by MERIT40 and TNKS1/2 work as scaffolds that control the DNA damage response (DDR) machinery and the spindle structure and function [37, 38].

\section{TNKS1/2 inhibitors in cancer}

Tankyrases have such a broad range of substrates, that their alteration (mutation, up- or downregulation) is related to multiple diseases, including obesity [85], diabetes, fibrosis [86], Epstein Barr and Herpes simplex viral infections [87, 88], Cherubism [17], sclerosis [89] and cancer. Through the analysis of the public cancer portal cBioportal we have obtained a profile of the main cancer types affected by alterations in TNKS $/ 2$ genes $[90,91]$. For both genes, endometrial is the one that presented the highest rate of TNKS and TNKS2 mutations (Fig. 3a and c), followed by colorectal cancer, bladder and esophagogastric (for TNKS) and melanoma, colorectal and prostate (for TNKS2, Fig. 3a and c). In the case of colorectal, bladder, hepatocellular and ovarian carcinoma alterations in TNKS expression are caused by deep deletion while in esophagogastric cancer TNKS gene amplification is responsible for most of the alterations in TNKS. Point mutations were responsible of TNKS2 alterations for most tumors with exception of prostate adenocarcinoma and B-cell neoplasms (Fig. 3a and c). The reasons for the differences in the mutation mechanisms affecting TNKS or TNKS2 differentially remain to be studied. Regarding the gene sequences, missense mutations were the most frequently found, followed by truncating, fusion and in-frame and they were distributed all along the genes (Fig. $3 \mathrm{~b}$ and d). Overall survival of patients presenting altered expression in TNKS or TNKS2 differed depending on the studies available in the public platform for both types of cancer, thus no conclusion can be drawn. High levels of TNKS1 and/or 


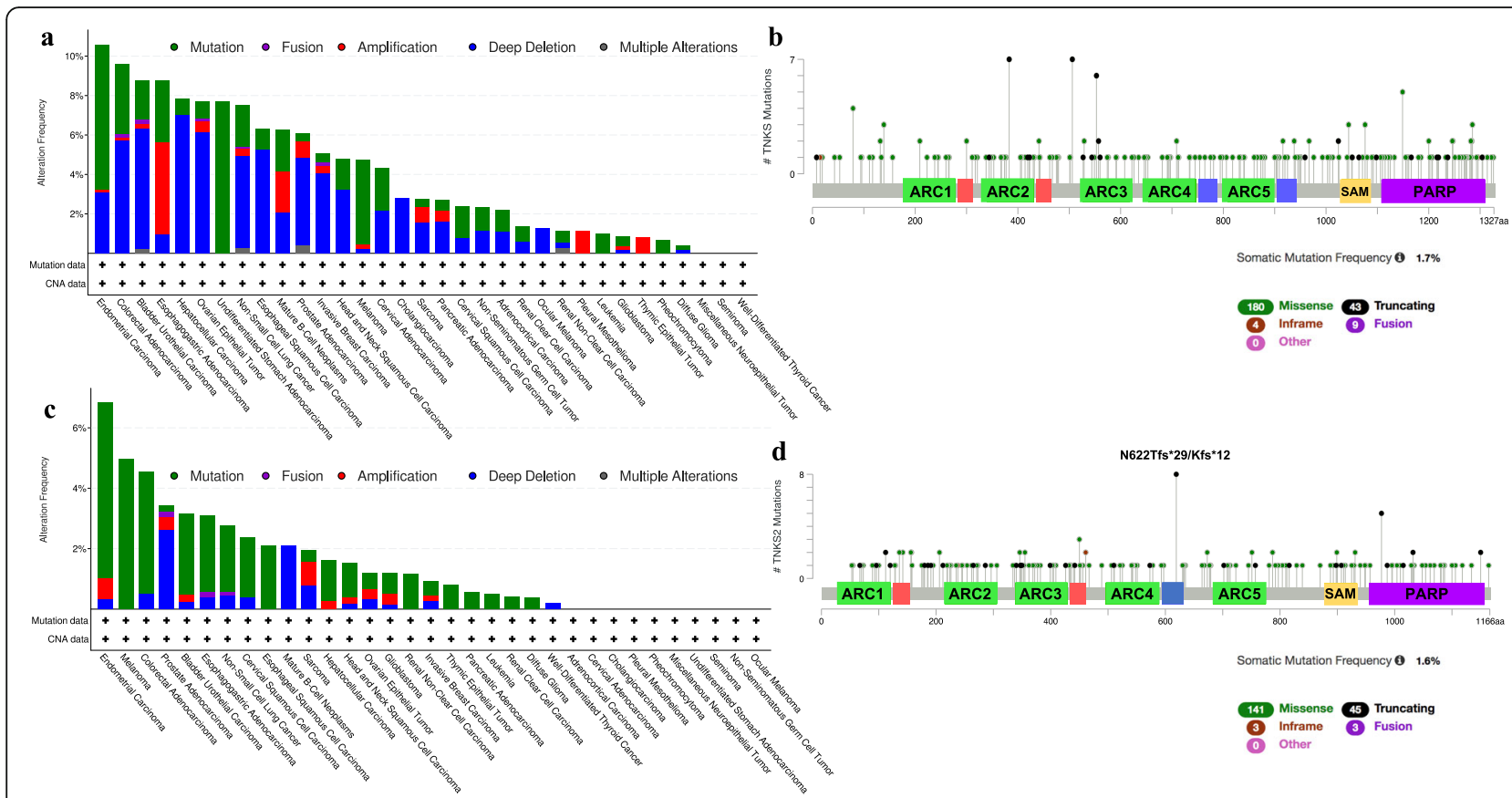

Fig. 3 TNKS1/2 alterations in cancer. The bar graphics show the percentage of alterations for TNKS and TNKS2 genes in multiple kinds of tumors ( $\mathbf{a}$ and $\mathbf{c}$ ) while the lollipop graphs show the mutation profile allong the sequence of both tankyrases genes (b and $\mathbf{d}$ ) according to the TCGA PanCancer Atlas Studies from the cBioportal website

TNKS2 expression have been observed mainly in colon $[92,93]$ and lung cancer $[39,94]$, but also in brain cancer [95], breast cancer [96], ovarian cancer [97] and liver cancer [98]. The first TNKS inhibitor XAV939 was discovered in 2009 by Huang and colleagues while they were looking into $\mathrm{Wnt} / \beta$-catenin pathway inhibitors [99]. Alterations in $\mathrm{Wnt} / \beta$-catenin signaling have been observed in different tumors but, there are no direct inhibitors against this pathway in clinical use. For this reason, several targets of the Wnt/ $\beta$-catenin pathway have been used to develop effective inhibitors. Porcupine inhibitors and anti-Frizzled antibodies, which block the secretion of Wnt ligands and prevent the ligand from binding to the receptor respectively, have been studied in clinical trials. Tankyrase inhibitors have been also developed as a therapeutic alternative to treat cancer, although there are no current clinical trials studying them [100-103]. These inhibitors recognize the catalytic domain and can be categorized according to the subsite of the donor $\mathrm{NAD}^{+}$site they target: nicotinamide subsite (NS) (XAV939, AZ1366, RK-582 and LZZ-02), adenosine subsite (AS) (IWR1, G007-LK, OD366, OM-1700 and K756) or dual binders (DB) if they recognize both nicotinamide and adenosine subsites (TNKS656) (Table 2). The nicotinamide subsite is highly conserved among the PARP family while the adenosine subsite is more specific of tankyrases. This fact allows those inhibitors that target adenosine subsite present high selectivity towards TNKS $[102,116]$. In the next section we will update the information about the role of tankyrases in cancer as well as the recent approaches using TNKS inhibitors as single and combined antitumor therapies. According to the current literature, colon and lung cancer have been two of the main models used for testing TNKS1/2 inhibitors (maybe due to the high percentage of alterations in the $\mathrm{Wnt} / \beta$-catenin signaling components). For this reason, we will tackle the information about the inhibitors from the point of view of the cancer

Table 2 Tankyrase-targeted anti-tumor options

\begin{tabular}{llll}
\hline TNKS inhibitor & Target & Type of cancer & References \\
\hline XAV939 & NS & Ovarian & {$[97]$} \\
& & Breast & {$[96,104]$} \\
& & Neuroblastoma & {$[105]$} \\
IWR1 & Hepatocellular & {$[98,106]$} \\
G007-LK & AS & Lung & {$[94]$} \\
& AS & Colon & {$[107]$} \\
& & Glioblastoma & {$[108]$} \\
OD366 & & Hepatocellular & {$[106]$} \\
OM-1700 & MS & Colanoma & {$[109]$} \\
K-756 & AS & Colon & {$[110]$} \\
AZ1366 & AS & Colon & {$[111]$} \\
TNKS656 & NS & Lung & {$[102]$} \\
& DB & Lung & {$[112]$} \\
RK-582 & & Hepatocellular & {$[113]$} \\
LZZ-02 & NS & Colon & {$[114]$} \\
\hline
\end{tabular}


model in which they have been tested, highlighting the achievements and the issues that arose after their use in each model.

\section{Colon carcinoma}

Colorectal cancer is the third most common cancer and the second leading cause of cancer death worldwide [117] . The Wnt/ $\beta$-catenin signaling pathway is hyperactivated in approximately $90 \%$ of human colorectal tumors most frequently due to the mutually exclusive mutation of tumor suppressor genes $A P C$ or $A X I N$ or of the CTNNB1 oncogene. These genetic alterations cause the accumulation of active $\beta$-catenin protein within the cell nucleus and the subsequent activation of its target genes that promote cell proliferation and malignancy [93, 101, 103, 115, 118]. Nowadays, TNKS inhibitors have gained attention due to its implication through the regulation of the limiting factor of the $\beta$-catenin degradation complex Axin [100, 102]. To date, many selective TNKS inhibitors with different structures and binding modes have been developed and used to treat colorectal cancer (Table 2).

G007-LK G007-LK binds specifically to the adenosine subsite of the TNKS catalytic domain $[119,120]$. This potent and selective inhibitor is able to induce the stabilization of Axin levels, which allows the stabilization of degradasomes in colon SW480 cell line. Degradasomes are structures formed by the components of $\beta$ catenin destruction complex which represent an active site for $\beta$-catenin turnover. The restoration of $\beta$-catenin degradation in APC-mutated cells after the treatment with G007-LK was shown, providing a link between TNKS inhibition and degradasomes formation in colorectal cancer cells [107]. Many new compounds have been synthesized from the chemical optimization of G007-LK. Two of them, OD366 and OM-1700 were deeply characterized and demonstrated good results and high selectivity towards TNKS1/2. Firstly, OD36 was obtained and showed a significant tumor size reduction after oral administration in the COLO 320DM xenograft immunodeficient mice model. Neither body weight loss nor animal discomforts was registered during the experimental period, although some adverse chemical properties required a new optimization, leading to OM-1700 identification. This new compound exhibited favorable parameters in general and reduced viability, proliferation and canonical Wnt $/ \beta$-catenin signaling through Axin $1 / 2$ stabilization and active $\beta$-catenin reduction, as well as, the downregulation of $\beta$-catenin target genes $[110,111]$.

K-756 This inhibitor was found to recognize the adenosine subsite of TNKS and stabilize and reduce Axin and active nuclear $\beta$-catenin levels, respectively. Besides, $\mathrm{K}$ -
756 was reported to induce cell growth inhibition in colon COLO 320DM and SW403 lines, whose proliferation is dependent on the $\mathrm{Wnt} / \beta$-catenin signaling due to a short truncating mutation in APC component. The efficacy of K-756 was shown in an oral-administered DLD-1/TCF-Luc colon cancer xenograft mice model, proving the antiproliferative activity of K-756 [102].

RK-582 RK-582 binds to the nicotinamide subsite of tankyrases with a 200 -fold selectivity against other PARP members and presents a remarkable level of efficacy at low doses. This new inhibitor blockades the Wnt/ $\beta$-catenin pathway by elevating Axin 2 and reducing active nuclear $\beta$-catenin levels while exhibits a potent tumor growth inhibitory effect in the mouse COLO-320DM xenograft model with oral or intraperitoneal administration $[101,115]$.

LZZ-02 The binding mode of LZZ-02 is similar to XAV939, since both recognize the nicotinamide pocket of TNKS. However, LZZ-02 forms more additional interactions with the residues near the border of the nicotinamide subsite in TNKS, which could improve the binding position and also enhance the inhibitory activity. LZZ-02 blockades the proliferation of APC mutated SW480 and DLD1 cells through the inhibition of the Wnt/ $/$-catenin signaling. In addition, this inhibitor showed a robust antitumor effect in a DLD1-derived colon tumor xenograft model, pointing out the promising therapeutic application [116].

Despite tankyrase inhibitors have shown multiple benefits both in vitro and in vivo models; there are still remaining issues that have to be elucidated. Firstly, the status of the APC mutation, which has been considered as a potential biomarker for sensitivity to TNKS inhibitors in colon cancer [121]. Most colorectal tumors contain truncated APC mutations. Although all the mutations trigger the same phenotype $(\mathrm{Wnt} / \beta$-catenin signaling hyperactivation), the mechanism underlying every mutation may cause different biochemical ways to respond to the same therapy [92]. According to the information obtained from K-756 and RK-287107, these inhibitors were able to induce cell growth inhibition in COLO-320DM and SW403 cells, but not in RKO, HCC2998 and DLD-1 cells. COLO-320DM and SW403 cell lines harbor "short-type" truncated APC mutations, which consist of lacking all the $\beta$-catenin-binding 20 amino acid repeats (20-AARs) and activate $\beta$-catenin signaling. Nevertheless, the other cell lines contain "longtype" truncated APC mutations, which partially preserve 20 -AARs and activate $\beta$-catenin signaling modestly [101, 102]. The reason that could explain the different effects of TNKS inhibitors between both types of mutations is that Long-type mutations have been reported to confer 
resistance to tankyrase inhibitors due to a dominant negative effect on Axin2-dependent $\beta$-catenin degradation [121]. However, a recent report proved that longtype truncated APC mutation cells are also sensitive to TNKS inhibitors [92, 93]. In fact, the recently discovered TNKS inhibitor LZZ-02 has shown a potent therapeutic effect in a xenograft model derived from the DLD-1 colon cells [116]. All this information points out the need to get representative genetic models to define the genetic context, understand the molecular mechanism of a signaling pathway as well as achieve an accurate and effective treatment. Other option could be the use of TNKS inhibitors as a combination therapy to enhance their antitumor effect in colorectal cancer models that are resistant to TNKS inhibition. Solberg and colleagues proposed the use of G007-LK at low dosage with the PI3K (BKM120) and the epidermal growth factor receptor (EGFR) (erlotinib) inhibitors to treat colon cancer. The information obtained suggested that the activity of G007-LK was potentiated by the inhibition of both PI3K and EGRF pathways in the TNKS inhibitor-sensitive COLO-320DM and the inhibitor-resistant HCT-15 cell lines in vitro and in vivo tumor xenografts models [122]. Another important issue related to TNKS inhibitors is the balance between efficacy and toxicity. G007-LK, one of the most characterized TNKS inhibitors, was found to exhibit a strong tumor growth inhibition in colon xenograft mouse models. However, the efficacy of G007-LK was limited by the intestinal toxicity that carried out to reduced crypt proliferation and epithelial degeneration in the small intestine of mice [120]. RK-582 displayed an optimal toxicity profile in a xenograft mouse model administered orally or intraperitoneally. Weight loss and general toxicity were not observed at moderate dosage, although modest levels of intestinal toxicity were appreciated at high dosage, pointing out the unavoidable intestinal toxicity of TNKS inhibitors at high doses suggested by Zhong and collaborators $[13,115]$. On the other hand, LZZ-02 was shown to have a potent antitumor effect as well as an extraordinary in vivo toxicity profile without body weight loss, which indicates that the inhibitor was well tolerated by mice [116]. These new inhibitors could become an effective cancer treatment, although the molecular mechanism requires additional investigation together with a better characterization in clinic.

\section{Lung cancer}

Lung cancer is the most diagnosed cancer and one of the foremost causes of cancer-associated mortality worldwide [123]. Non-small cell lung cancer (NSCLC) is the most common type of lung cancer, which encompasses around $80 \%$ of these cases [112]. Unlike colon carcinoma, abnormal Wnt activity in lung cancer is not only associated with APC or $\beta$-catenin mutations, but also a deregulation in the expression of genes associated with the canonical and non-canonical Wnt pathway. It has been found different components such upstream Wnt signaling effectors, for instance Dishvelled 3 (Dvl3 ), or downregulation of Wnt antagonists as Wntinhibitory factor 1 (WIF 1) [94, 124]. In addition, TNKS1 and TNKS2 levels are elevated in lung cancer, as well as $\beta$-catenin levels [94, 123]. Another relevant pathway associated with some types of cancer is the Hippo-YAP (yes-associated-protein) pathway. It has been reported that alterations due to the activation in the Hippo kinase cascade trigger the phosphorylation of YAP, which is sequestered in the cytoplasm. When this cascade is not active, YAP can enter the nucleus and activate a downstream transcriptional program by the binding to TEAD family transcription factors. If this pathway is highly activated, it gives rise to an uncontrolled cell proliferation [113]. The effect of several TNKS inhibitors has been assessed in human and mice lung models (Table 2).

IWR1 IWR-1 has the ability to inhibit TNKS binding the adenosine binding pocket with a specificity of 100fold than XAV939 for TNKS enzymes over other PARPs $[99,125]$. The use of this inhibitor in human and murine lung cancer cell lines have provided a reduction in cell growth, as well as a decrease in the Wnt pathway, stabilizing protein levels of Axin and TNKS1/2 [94].

AZ1366 AZ1366 occupies the nicotinamide binding site of the PARP catalytic domain and works stabilizing Axin1 and decreasing the mRNA levels of different $\beta$ catenin targets. The most common subtype of NSCLC is associated with activating mutations in the EGFR gene. AZ1366 has shown a synergistic effect suppressing NSCLC proliferation in combination with EGFR inhibitors. When analyzing Wnt-responsive tumors in mice, an improvement in tumor control and survival using this combined inhibition was found compared to the single use of EGFR inhibitors [112].

TNKS656 This inhibitor is based on the optimization of XAV939, although it is consider a dual binder (DB) inhibitor since it binds both nicotinamide and adenoside subsites [126]. The efficacy of TNKS656 was demonstrated through the downregulation of nuclear YAP expression. This effect over YAP signaling is due to the angiomotin family proteins, which are tankyrase substrates. TNKS656 also proved a synergic effect with EGFR inhibitors in PC9, HCC827 and HCC4006 NSCLC cell lines through the inhibition of YAP signaling [113, 126, 127]. 


\section{Other tumors}

The role of tankyrases in the development of colon and lung cancer and the use of TNKS inhibitors are better known. Nevertheless, there is a growing knowledge concerning the use of TNKS inhibitors in other types of cancer (Table 2). The TNKS inhibitor XAV939 has been tested in ovarian cancer [97], breast cancer [96, 104], neuroblastoma [105] and hepatocellular carcinoma [98, 106]. It has been observed in these tumors that the use of XAV939 attenuates cell proliferation, viability by induction of apoptosis, migration, invasion and metastasis, as well as a decrease in aerobic glycolysis rate, which is one of the hallmarks of cancer. Besides, the use of XAV939 with the Polo-Like Kinase 1 (PLK1) inhibitor shown a synergistic effect concerning invasion, migration and proliferation, demonstrating the importance of PLK1 for TNKS1 stability [104]. The benefits of G007LK have been reported in glioblastoma, proving a reduction in cell proliferation and sphere formation [108]. A decrease in cell growth and proliferation was also shown in hepatocellular carcinoma after the treatment of G007LK combined with MEK and Akt inhibitors [106]. Moreover, the significance of the $\mathrm{Wnt} / \beta$-catenin pathway and YAP signalling in the immune evasion has led to a combinatorial therapeutic strategy with the immune checkpoint inhibitor anti-PD-1 and G007-LK in melanoma, demonstrating the capacity to sensitize the tumor to the immune checkpoint therapy [109]. Recently, studies with the TNKS656 inhibitor in hepatocarcinoma cell lines gave evidences of suppression in cell proliferation, along with reduction in the levels of epithelial-mesenchymal transition (EMT) markers, invasion and metastasis [114].

\section{New TNKS inhibitors targeting the ANK domain}

All the current TNKS inhibitors are targeting the catalytic PARP domain. Tankyrase catalytic inhibition triggers the accumulation of many substrates and also TNKS1/2 [23]. Recently, it has been described a new catalysis-independent role of tankyrases as scaffolding proteins and the accumulation of tankyrases and their binding partners could promote the scaffolding mechanisms of both tankyrases [14, 31]. For this reason it has been explored different ways to develop new TNKS inhibitors against the ANK domain, which is responsible for the scaffolding function through the recognition and binding to the substrates of tankyrase. The strategy based on the binding mode of the fourth ARC of TNKS2 with the TBM of 3BP2 developed macrocyclized peptides that mimic the natural binding partners. Two of these peptides were able to disrupt the interaction with the substrates in vitro, and further showed the ability to enter cells and suppress the Wnt/ $\beta$-catenin signaling through the disruption of TNKS-Axin interaction [128]. Another method based on a virtual screening was performed in order to find a molecule that could interrupt the interaction between tankyrase and the ubiquitin-specific protease 25 (USP25), which previously was shown to deubiquitinate and stabilize TNKS1/2 [129]. C44 was found to act as a protein-protein interaction (PPi) inhibitor that promoted the degradation of TNKS1/2, contributing to the inhibition of Wnt/3-catenin signaling via Axin stabilization and thus $\beta$-catenin degradation. C44 effectively decreased cell proliferation in the prostate cancer cell line PC-3, as well as it reduced cancer tumor growth in xenograft mice models [130]. Nowadays, two other different methods have been developed to find new peptidomimetics binding the ANK domain of tankyrase. One of them consists in a fragment-based screening using a combination of differential scanning fluorimetry (DSF) and nuclear magnetic resonance (NMR) spectroscopy [131]. The second one is an in vitro assay based on a fluorescence resonance energy transfer (FRET), which represents a reliable and cost-effective method to test compounds inhibiting the scaffolding function of tankyrase. Two molecules binding the ARC4 of TNKS2 with significant affinity were obtained using this method, indicating the potential therapeutic relevance of this new approach to find effective TNKS inhibitors [132].

\section{Conclusions}

In view of the current scientific literature, there is still a long way to go to study in a more precise and systematic manner the implications of TNKS1/2 in the development of cancer. The role of tankyrases in the optimization of the Wnt/ $\beta$-catenin pathway, key in the development of colorectal cancer and with implications in numerous types of cancer, is clear. Therefore, it is necessary to consolidate clinical studies and global data analysis to improve our current view of the role of TNKS1/2 in the origin of the alterations that lead to tumor development and the use of new therapeutic agents based on inhibition by tankyrases. In addition, it will be necessary in the future to deepen the knowledge of the TNKS interactoma in normal and tumor cells to define and identify new TNKS substrates. Future investigations will be also needed to elucidate the new functions of tankyrases that will allow to desing pharmacological inhibitors disrupting the TBM-ARC interaction independtly of the poly (ADP-ribosylation) activity.

\section{Abbreviations}

3BP2: SH3 domain binding protein 2; AAR: Amino acid repeat; Akt: Protein kinase B; AMPK: AMP-activated protein kinase; ANK: Ankyrin;

APC: Adenomatous polyposis coli; ARC: Ankyrin repeat cluster; ARTD: ADP ribosyl transferases diphtheria toxin-like; AS: Adenosine subsite;

ATG9: Autophagy-related protein 9A; Axin1/2: Axis inhibition 1/2; BCl-2: B-cell lymphoma 2; BRCA1/2: Breast cancer 1/2; CK1a: Casein-kinase 1 alpha;

CTNNB1: Catenin Beta 1; DDR: DNA Damage Response; DSF: Differential 
scanning fluorimetry; DB: Dual binder; Dvl-3: Dishvelled; EGFR: Epidermal growth factor receptor; EMT: Epithelial-mesenchymal transition; FDA: Food and Drug Administration; FRET: Fluorescence resonance energy transfer; GMD: GDP-Mannose-4,6-Dehydratase; GSK3ß: Glycogen synthase kinase 3B; HPS: His, Pro and Ser; IF4A1: Eukaryotic translation initiation factor 4A1; LKB1: Liver kinase B1; MCl-1: Myeloid cell leukemia-1; MEK: Mitogen-activated protein kinase kinase; MERIT40: Mediator of Rap80 Interactions and Targeting 40 kD; MO25: Mouse protein 25; NAD: Nicotinamide adenine dinucleotide; NELFE: Negative elongation factor complex member e; NMR: Nuclear magnetic resonance; NS: Nicotinamide subsite; NSCLC: Non-small cell lung cancer; PAR: Poly (ADP-ribose); PARdU: PAR-dependent ubiquitination; PARP: Poly (ADP-ribose) polymerase; PD-1: Programmed cell death-1; PEX14: Peroxin-14; PEX5: Peroxin-5; PI3K: Phosphatidylinositol 3-kinase; PLK1: Polo-Like Kinase 1; Pol-- $\beta$ : Polimerase-beta; PPi: Protein-protein interaction; PTEN: Phosphatidylinositol $(3,4,5)$-trisphosphate phosphatase; PTM: Posttranslational modification; RING: Really interesting new gene; RNF146: RING finger protein 146; SA1: Stromal antigen 1; SAM: Sterile alpha module; SCF: Skp, Cullin, F-box containing complex; SSB: Single Strand Break; SSBR: Single Strand Break Repair; STRAD: STE20 Related Adaptor Alpha; SUMO: Small Ubiquitin-like Modifier; TBM: Tankyrase binding motif; TCF: T cell factor; TEAD: Transcriptional enhanced associate domain; TIN2: TRF1interactin nuclear protein 2; TNKS: Tankyrase; TRF1: Telomeric repeat-binding factor 1; USP25: Ubiquitin-specific protease 25; WIF 1: Wnt-inhibitory factor 1; WWE: Trp, Trp, Glu; XRCC1: X-ray repair crosscomplementing protein 1; YAP: Yes-associated-protein; $\beta$-TRCP: Beta-transducin repeats-containing proteins

\section{Acknowledgements}

Not applicable.

\section{Authors' contributions}

Writing-Review \& Editing: EZ. Writing-Review, ABH, AM. Supervision and conceptualization: JMRV and FJO. All authors read and approved the final manuscript.

\section{Authors' information}

Esteban Zamudio-Martinez, José Manuel Rodríguez-Vargas, F. Javier Oliver Instituto de Parasitología y Biomedicina López Neyra, CSIC, Granada, Spain, CIBERONC, Centro de Investigación Biomédica en Red de Cáncer (CIBERONC), 28029 Madrid, Spain.

Alberto Muñoz, Centro de Investigación Biomédica en Red de Cáncer (CIBERONC), 28029 Madrid, Spain, and Instituto de Investigaciones Biomédicas "Alberto Sols", Consejo Superior de Investigaciones Científicas, Universidad Autónoma de Madrid, 28029 Madrid, Spain. Ana Belén Herrera-Campos, Instituto de Parasitología y Biomedicina López Neyra, CSIC, Granada, Spain.

\section{Funding}

The work in the authors' laboratory is funded by the Agencia Estatal de Investigación SAF2012-40011-C02-01, SAF2015-70520-R, RTI2018-098968-BI00, RTICC RD12/0036/0026 and PID2019-104867RB-I00/AEI/https://doi.org/10. 13039/501100011033), and Instituto de Salud Carlos III-Fondo Europeo de Desarrollo Regional (CIBERONC ISCIII CB16/12/00421 and CB16/12/00273); by Junta de Andalucía, project of Excellence from Junta de Andalucía P10-CTS0662, P12-CTS-383 to FJO, CIBER Cáncer to FJO. Fundación Domingo Martínez (call 2019). JMRV is funded through a contract Juan de la Cierva (Reincorporación) from Ministerio de Ciencia e Innovación.

\section{Availability of data and materials}

Not applicable.

\section{Declarations}

Ethics approval and consent to participate Not applicable.

\section{Consent for publication}

Not applicable.

\section{Competing interests}

The authors declare that they have no competing interests.

\section{Author details}

'Instituto de Parasitología y Biomedicina López Neyra, CSIC, CIBERONC, 18016 Granada, Spain. ${ }^{2}$ Centro de Investigación Biomédica en Red de Cáncer, CIBERONC, 28029 Madrid, Spain. ${ }^{3}$ Instituto de Investigaciones Biomédicas "Alberto Sols", CSIC, Universidad Autónoma de Madrid, 28029 Madrid, Spain.

Received: 7 February 2021 Accepted: 15 April 2021

Published online: 28 April 2021

\section{References}

1. Hottiger MO, Hassa PO, Lüscher B, Schüler H, Koch-Nolte F. Toward a unified nomenclature for mammalian ADP-ribosyltransferases. Trends Biochem Sci. 2010;35(4):208-19. https://doi.org/10.1016/j.tibs.2009.12.003.

2. Palazzo L, Mikoč A, Ahel I. ADP-ribosylation: new facets of an ancient modification. FEBS J. 2017;284(18):2932-46. https://doi.org/10.1111/febs.14 078.

3. Kaminker PG, Kim SH, Taylor RD, Zebarjadian Y, Funk WD, Morin GB, et al. TANK2, a new TRF1-associated poly (ADP-ribose) polymerase, causes rapid induction of cell death upon overexpression. J Biol Chem. 2001;276(38): 35891-9.

4. Smith S. The world according to PARP. Trends Biochem Sci. 2001;26:174-9.

5. Hsiao SJ, Smith S. Tankyrase function at telomeres, spindle poles, and beyond. Biochimie. 2008;90:83-92.

6. Eisemann T, McCauley M, Langelier MF, Gupta K, Roy S, Van Duyne GD, et al. Tankyrase-1 Ankyrin repeats form an adaptable binding platform for targets of ADP-ribose modification. Structure. 2016;24(10):1679-92. Available from:. https://doi.org/10.1016/j.str.2016.07.014.

7. Min A, Im SA. PARP inhibitors as therapeutics: Beyond modulation of parylation. Cancers (Basel). 2020;12(2):394.

8. Martí JM, Fernández-Cortés M, Serrano-Sáenz S, Zamudio-Martinez E, Delgado-Bellido D, Garcia-Diaz A, et al. The multifactorial role of PARP-1 in tumor microenvironment. Cancers (Basel). 2020;12(3):739.

9. Kim MK. Novel insight into the function of Tankyrase (review). Oncol Lett. 2018;16(6):6895-902. https://doi.org/10.3892/ol.2018.9551.

10. Smith S, De Lange T. Tankyrase promotes telomere elongation in human cells. Curr Biol. 2000;10(20):1299-302. https://doi.org/10.1016/S0960-9822 (00)00752-1

11. Mariotti L, Pollock K, Guettler S. Regulation of Wnt/ß-catenin signalling by tankyrase-dependent poly (ADP-ribosyl) ation and scaffolding. $\mathrm{Br} J$ Pharmacol. 2017:174(24):4611-36. https://doi.org/10.1111/bph.14038.

12. Canudas S, Houghtaling BR, Kim JY, Dynek JN, Chang WG, Smith S. Protein requirements for sister telomere association in human cells. EMBO J. 2007; 26(23):4867-78. https://doi.org/10.1038/sj.emboj.7601903.

13. Zhong L, Ding Y, Bandyopadhyay G, Waaler J, Börgeson E, Smith S, et al. The PARsylation activity of tankyrase in adipose tissue modulates systemic glucose metabolism in mice. Diabetologia. 2016;59(3):582-91. https://doi. org/10.1007/s00125-015-3815-1.

14. Li X, Han H, Zhou MT, Yang B, Ta AP, Li N, et al. Proteomic analysis of the human Tankyrase protein interaction network reveals its role in Pexophagy. Cell Rep. 2017;20(3):737-49. https://doi.org/10.1016/j.celrep.2017.06.077.

15. Yang L, Sun L, Teng Y, Chen H, Gao Y, Levine AS, et al. Tankyrase1-mediated poly (ADP-ribosyl) ation of TRF1 maintains cell survival after telomeric DNA damage. Nucleic Acids Res. 2017;45(7):3906-21. https://doi.org/10.1093/nar/ gkx083.

16. Langelier MF, Eisemann T, Riccio AA, Pascal JM. PARP family enzymes: regulation and catalysis of the poly (ADP-ribose) posttranslational modification. Curr Opin Struct Biol. 2018;53:187-98. https://doi.org/10.1016/j. sbi.2018.11.002.

17. Guettler S, Larose J, Petsalaki E, Gish G, Scotter A, Pawson T, et al. Structural basis and sequence rules for substrate recognition by tankyrase explain the basis for cherubism disease. Cell. 2011;147(6):1340-54. https://doi.org/10.101 6/j.cell.2011.10.046.

18. De Rycker M, Venkatesan RN, Wei C, Price CM. Vertebrate tankyrase domain structure and sterile a motif (SAM)-mediated multimerization. Biochem J. 2003;372(1):87-96. https://doi.org/10.1042/bj20021450.

19. Seimiya $\mathrm{H}$, Smith $\mathrm{S}$. The telomeric poly (ADP-ribose) polymerase, tankyrase 1 , contains multiple binding sites for telomeric repeat binding factor 1 (TRF1) and a novel acceptor, 182-kDa tankyrase-binding protein (TAB182). J Biol Chem. 2002;277(16):14116-26. https://doi.org/10.1074/jbc.M112266200. 
20. Azarm K, Smith S. Nuclear PARPs and genome integrity. Genes Dev. 2020; 34(5-6):285-301. https://doi.org/10.1101/gad.334730.119.

21. Haikarainen T, Krauss S, Lehtio L. Tankyrases: structure, function and therapeutic implications in Cancer. Curr Pharm Des. 2014;20(41):6472-88. https://doi.org/10.2174/1381612820666140630101525.

22. Chi NW, Lodish HF. Tankyrase is a Golgi-associated mitogen-activated protein kinase substrate that interacts with IRAP in GLUT4 vesicles. J Biol Chem. 2000;275(49):38437-44. https://doi.org/10.1074/jbc.M007635200.

23. Bhardwaj A, Yang Y, Ueberheide B, Smith S. Whole proteome analysis of human tankyrase knockout cells reveals targets of tankyrase-mediated degradation. Nat Commun. 2017;8(1):2214

24. Smith S, Giriat I, Schmitt A, De Lange T. Tankyrase, a poly (ADP-ribose) polymerase at human telomeres. Science (80- ). 1998;282(5393):1484-7.

25. Smith S, De Lange T. Cell cycle dependent localization of the telomeric PARP, tankyrase, to nuclear pore complexes and centrosomes. J Cell Sci. 1999;112(21):3649-55.

26. DaRosa PA, Klevit RE, Xu W. Structural basis for tankyrase-RNF146 interaction reveals noncanonical tankyrase-binding motifs. Protein Sci. 2018;27(6):105767. https://doi.org/10.1002/pro.3413.

27. Sbodio Jl, Chi NW. Identification of a tankyrase-binding motif shared by IRAP, TAB182, and human TRF1 but not mouse TRF1: NuMA contains this RXXPDG motif and is a novel tankyrase partner. J Biol Chem. 2002;277(35): 31887-92. https://doi.org/10.1074/jbc.M203916200.

28. Cook BD, Dynek JN, Chang W, Shostak G, Smith S. Role for the related poly (ADP-ribose) polymerases Tankyrase 1 and 2 at human telomeres. Mol Cell Biol. 2002;22(1):332-42. https://doi.org/10.1128/MCB.22.1.332-342.2002.

29. Fan C, Yarravarapu N, Chen H, Kulak O, Dasari P, Herbert J, et al. Regulation of tankyrase activity by a catalytic domain dimer interface. Biochem Biophys Res Commun. 2018;503(3):1780-5. https://doi.org/10.1016/j.bbrc.2018.07.113.

30. De Rycker M, Price CM. Tankyrase polymerization is controlled by its sterile alpha motif and poly (ADP-ribose) polymerase domains. Mol Cell Biol. 2004; 24(22):9802-12. https://doi.org/10.1128/MCB.24.22.9802-9812.2004.

31. Mariotti L, Templeton CM, Ranes M, Paracuellos P, Cronin N, Beuron F, et al. Tankyrase requires SAM domain-dependent polymerization to support Wnt$\beta$-catenin signaling. Mol Cell. 2016;63(3):498-513. Available from:. https://doi. org/10.1016/j.molcel.2016.06.019.

32. Eisemann T, Langelier MF, Pascal JM. Structural and functional analysis of parameters governing tankyrase-1 interaction with telomeric repeat-binding factor 1 and GDP-mannose 4,6-dehydratase. J Biol Chem. 2019;294(40): 14574-90. https://doi.org/10.1074/jbc.RA119.009200.

33. DaRosa PA, Wang Z, Jiang X, Pruneda JN, Cong F, Klevit RE, et al. Allosteric activation of the RNF146 ubiquitin ligase by a poly (ADP-ribosyl) ation signal. Nature. 2014;517(7533):223-6.

34. Li N, Zhang Y, Han X, Liang K, Wang J, Feng L, et al. Poly-ADP ribosylation of PTEN by tankyrases promotes PTEN degradation and tumor growth. Genes Dev. 2015;29(2):157-70. https://doi.org/10.1101/gad.251785.114.

35. Bae J, Donigian JR, Hsueh AJW. Tankyrase 1 interacts with Mcl-1 proteins and inhibits their regulation of apoptosis. J Biol Chem. 2003;278(7):5195204. https://doi.org/10.1074/jbc.M201988200.

36. Bisht KK, Dudognon C, Chang WG, Sokol ES, Ramirez A, Smith S. GDPMannose-4,6-Dehydratase is a cytosolic partner of Tankyrase 1 that inhibits its poly (ADP-ribose) polymerase activity. Mol Cell Biol. 2012;32(15):3044-53.

37. Okamoto K, Ohishi T, Kuroiwa M, lemura SI, Natsume T, Seimiya H. MERIT40dependent recruitment of tankyrase to damaged DNA and its implication for cell sensitivity to DNA-damaging anticancer drugs. Oncotarget. 2018; 9(88):35844-55. https://doi.org/10.18632/oncotarget.26312.

38. Zheng D, Xie W, Li L, Jiang W, Zou Y, Chiang C, et al. RXXPEG motif of MERIT40 is required to maintain spindle structure and function through its interaction with Tankyrase1. Cell Biol Int. 2019;43(2):174-81. https://doi.org/10.1002/cbin.11086.

39. Li N, Wang Y, Neri S, Zhen Y, Fong LWR, Qiao Y, et al. Tankyrase disrupts metabolic homeostasis and promotes tumorigenesis by inhibiting LKB1AMPK signalling. Nat Commun. 2019;10(1):4363.

40. Callow MG, Tran H, Phu L, Lau T, Lee J, Sandoval WN, et al. Ubiquitin ligase RNF146 regulates tankyrase and Axin to promote Wnt signaling. PLoS One. 2011;6(7):e22595.

41. Zhang Y, Liu S, Mickanin C, Feng Y, Charlat O, Michaud GA, et al. RNF146 is a poly (ADP-ribose)-directed E3 ligase that regulates axin degradation and Wnt signalling. Nat Cell Biol [Internet]. 2011;13(5):623-9. Available from:. https://doi.org/10.1038/ncb2222.

42. He F, Tsuda K, Takahashi M, Kuwasako K, Terada T, Shirouzu M, et al. Structural insight into the interaction of ADP-ribose with the PARP WWE domains. FEBS Lett. 2012;586(21):3858-64. Available from:. https://doi.org/1 0.1016/j.febslet.2012.09.009

43. Vivelo CA, Ayyappan V, Leung AKL. Poly (ADP-ribose)-dependent ubiquitination and its clinical implications. Biochem Pharmacol. 2019;167:312. Available from. https://doi.org/10.1016/j.bcp.2019.05.006.

44. Li B, Qiao R, Wang Z, Zhou W, Li X, Xu W, et al. Crystal structure of a tankyrase 1-telomere repeat factor 1 complex. Acta Crystallogr Sect Struct Biol Commun. 2016;72(4):320-7. https://doi.org/10.1107/S2053230X1 6004131.

45. Dynek JN, Smith S. Resolution of Sister Telomere Association Is Required for Progression Through Mitosis. Science (80- ). 2004;304(5667):97-100.

46. Kang HC, Lee Yl, Shin JH, Andrabi SA, Chi Z, Gagné JP, et al. Iduna is a poly (ADP-ribose) (PAR)-dependent E3 ubiquitin ligase that regulates DNA damage. Proc Natl Acad Sci U S A. 2011;108(34):14103-8. https://doi.org/1 0.1073/pnas.1108799108.

47. Zhou ZD, Chan CHS, Xiao ZC, Tan EK. Ring finger protein 146/lduna is a poly (ADP-ribose) polymer binding and PARsylation dependent E3 ubiquitin ligase. Cell Adhes Migr. 2011;5(6):463-71. https://doi.org/10.4161/cam.5.6.183 56.

48. Wang Z, Michaud GA, Cheng Z, Zhang Y, Hinds TR, Fan E, et al. Recognition of the iso-ADP-ribose moiety in poly (ADP-ribose) by WWE domains suggests a general mechanism for poly (ADP-ribosyl)ation-dependent ubiquitination. Genes Dev. 2012;26(3):235-40. https://doi.org/10.1101/gad.1 82618.111.

49. Lüscher B, Bütepage M, Eckei L, Krieg S, Verheugd P, Shilton BH. ADPRibosylation, a multifaceted posttranslational modification involved in the control of cell physiology in health and disease. Chem Rev. 2018;118(3): 1092-136. https://doi.org/10.1021/acs.chemrev.7b00122.

50. Morrone S, Cheng Z, Moon RT, Cong F, Xu W. Crystal structure of a tankyrase-axin complex and its implications for axin turnover and tankyrase substrate recruitment. Proc Natl Acad Sci U S A. 2012;109(5):1500-5. https:// doi.org/10.1073/pnas.1116618109.

51. Thorvaldsen TE, Pedersen NM, Wenzel EM, Stenmark H. Differential roles of AXIN1 and AXIN2 in tankyrase inhibitor-induced formation of degradasomes and $\beta$-catenin degradation. PLoS One. 2017;12(1):1-12.

52. Krishnamurthy $N$, Kurzrock R. Targeting the Wnt/beta-catenin pathway in cancer: update on effectors and inhibitors. Cancer Treat Rev. 2018;62:50-60. Available from. https://doi.org/10.1016/j.ctrv.2017.11.002.

53. Maccario H, Perera NM, Davidson L, Downes CP, Leslie NR. PTEN is destabilized by phosphorylation on Thr366. Biochem J. 2007;405(3):439-44. https://doi.org/10.1042/BJ20061837.

54. Chen CY, Chen J, He L, Stiles BL. PTEN: Tumor suppressor and metabolic regulator. Front Endocrinol (Lausanne). 2018;9:1-12.

55. Manning BD, Cantley LC. AKT/PKB Signaling: Navigating Downstream. Cell. 2007;129:1261-74.

56. Wang $X$, Jiang $X$. Post-translational regulation of PTEN. Oncogene. 2008; 27(41):5454-63.

57. Okumura K, Mendoza M, Bachoo RM, DePinho RA, Cavenee WK, Furnari FB. PCAF modulates PTEN activity. J Biol Chem. 2006;281(36):26562-8. https:// doi.org/10.1074/jbc.M605391200.

58. Bassi C, Ho J, Srikumar T, Dowling RJO, Gorrini C, Miller SJ, et al. Nuclear PTEN controls DNA repair and sensitivity to genotoxic stress. Science (80- ). 2013;341(6144):395-9.

59. Vazquez F, Ramaswamy S, Nakamura N, Sellers WR. Phosphorylation of the PTEN tail regulates protein stability and function. Mol Cell Biol. 2000;20(14): 5010-8. https://doi.org/10.1128/MCB.20.14.5010-5018.2000.

60. Kullmann L, Krahn MP. Controlling the master - upstream regulation of the tumor suppressor LKB1. Oncogene. 2018;37(23):3045-57. Available from: https://doi.org/10.1038/s41388-018-0145-z.

61. Woods A, Johnstone SR, Dickerson K, Leiper FC, Fryer LGD, Neumann D, et al. LKB1 is the upstream kinase in the AMP-activated protein kinase Cascade. Curr Biol. 2003;13(22):2004-8. https://doi.org/10.1016/j.cub.2003.10. 031.

62. Fullerton MD, Galic S, Marcinko K, Sikkema S, Pulinilkunnil T, Chen ZP, et al. Single phosphorylation sites in Acc1 and Acc2 regulate lipid homeostasis and the insulin-sensitizing effects of metformin. Nat Med. 2013;19(12):164954. https://doi.org/10.1038/nm.3372.

63. Faubert B, Vincent EE, Griss T, Samborska B, Izreig S, Svensson RU, et al. Loss of the tumor suppressor LKB1 promotes metabolic reprogramming of cancer cells via HIF-1a. Proc Natl Acad Sci U S A. 2014;111(7):2554-9. https://doi.org/10.1073/pnas.1312570111. 
64. Gwinn DM, Shackelford DB, Egan DF, Mihaylova MM, Mery A, Vasquez DS, et al. AMPK phosphorylation of raptor mediates a metabolic checkpoint. Mol Cell. 2008;30(2):214-26. https://doi.org/10.1016/j.molcel.2008.03.003.

65. Deng M, Yang X, Qin B, Liu T, Zhang H, Guo W, et al. Deubiquitination and activation of AMPK by USP10. Mol Cell. 2016;61(4):614-24. https://doi.org/1 0.1016/j.molcel.2016.01.010

66. Hardie DG, Ross FA, Hawley SA. AMPK: a nutrient and energy sensor that maintains energy homeostasis. Nat Rev Mol Cell Biol. 2012;13(4):251-62. https://doi.org/10.1038/nrm3311.

67. Zeqiraj E, Filippi BM, Deak M, Alessi DR, Van Aalten DMF. Structure of the LKB1-STRAD-MO25 complex reveals an allosteric mechanism of kinase activation. Science (80- ). 2009;326(5960):1707-11.

68. Baas AF, Boudeau J, Sapkota GP, Smit L, Medema R, Morrice NA, et al. Activation of the tumour suppressor kinase LKB1 by the STE20-like pseudokinase STRAD. EMBO J. 2003;22(12):3062-72. https://doi.org/10.1093/ emboj/cdg292.

69. Boudeau J, Baas AF, Deak M, Morrice NA, Kieloch A, Schutkowski M, et al. $\mathrm{MO} 25 \mathrm{a} / \beta$ interact with STRADa/ $\beta$ enhancing their ability to bind, activate and localize LKB1 in the cytoplasm. EMBO J. 2003;22(19):5102-14. https:// doi.org/10.1093/emboj/cdg490

70. Xie Z, Dong Y, Scholz R, Neumann D, Zou MH. Phosphorylation of LKB1 at serine 428 by protein kinase $C-\zeta$ is required for metformin-enhanced activation of the AMP-activated protein kinase in endothelial cells. Circulation. 2008;117(7):952-62. https://doi.org/10.1161/CIRCULATIONAHA.1 07.744490 .

71. Ohyama C, Smith PL, Angata K, Fukuda MN, Lowe JB, Fukuda M. Molecular cloning and expression of GDP-D-mannose-4,6-dehydratase, a key enzyme for fucose metabolism defective in Lec13 cells. J Biol Chem. 1998;273(23): 14582-7. https://doi.org/10.1074/jbc.273.23.14582.

72. Becker DJ, Lowe JB. Fucose: Biosynthesis and biological function in mammals. Glycobiology. 2003;13(7):4363.

73. Schneider M, Al-Shareffi E, Haltiwanger RS. Biological functions of fucose in mammals. Glycobiology. 2017;27(7):601-18. https://doi.org/10.1093/glycob/ cwX034.

74. Michels J, Johnson PWM, Packham G. Mcl-1. Int J Biochem Cell Biol. 2005; 37(2):267-71. https://doi.org/10.1016/j.biocel.2004.04.007.

75. De Blasio A, Vento R, Di Fiore R. Mcl-1 targeting could be an intriguing perspective to cure cancer. J Cell Physiol. 2018;233(11):8482-98. https://doi. org/10.1002/jcp.26786.

76. DaRosa PA, Ovchinnikov S, Xu W, Klevit RE. Structural insights into SAM domain-mediated tankyrase oligomerization. Protein Sci. 2016;25(9):1744-52. https://doi.org/10.1002/pro.2968.

77. Riccio AA, McCauley M, Langelier MF, Pascal JM. Tankyrase sterile a motif domain polymerization is required for its role in Wnt signaling. Structure. 2016;24(9):1573-81. https://doi.org/10.1016/j.str.2016.06.022

78. Kim PK, Hailey DW, Mullen RT, Lippincott-Schwartz J. Ubiquitin signals autophagic degradation of cytosolic proteins and peroxisomes. Proc Natl Acad Sci U S A. 2008;105(52):20567-74. https://doi.org/10.1073/pnas.0810611105.

79. Nazarko TY. Pexophagy is responsible for $65 \%$ of cases of peroxisome biogenesis disorders. Autophagy. 2017;13(5):991-4. Available from:. https:// doi.org/10.1080/15548627.2017.1291480

80. Cho DH, Kim YS, Jo DS, Choe SK, Jo EK. Pexophagy: molecular mechanisms and implications for health and diseases. Mol Cells. 2018;41(1):55-64. https://doi.org/10.14348/molcells.2018.2245.

81. Brocard C, Lametschwandtner G, Koudelka R, Hartig A. Pex $14 p$ is a member of the protein linkage map of Pex5p. EMBO J. 1997;16(18):5491-500. https:// doi.org/10.1093/emboj/16.18.5491.

82. Van Zutphen T, Veenhuis M, Van Der Klei IJ. Pex14 is the sole component of the peroxisomal translocon that is required for pexophagy. Autophagy. 2008;4(1):63-6. https://doi.org/10.4161/auto.5076.

83. Orsi A, Razi M, Dooley HC, Robinson D, Weston AE, Collinson LM, et al. Dynamic and transient interactions of Atg9 with autophagosomes, but not membrane integration, are required for autophagy. Mol Biol Cell. 2012; 23(10):1860-73. https://doi.org/10.1091/mbc.e11-09-0746.

84. Zavodszky E, Vicinanza M, Rubinsztein DC. Biology and trafficking of ATG9 and ATG16L1, two proteins that regulate autophagosome formation. FEBS Lett. 2013;587(13):1988-96. https://doi.org/10.1016/j.febslet.2013.04.025.

85. Wang H, Kuusela S, Rinnankoski-Tuikka R, Dumont V, Bouslama R, Ramadan UA, et al. Tankyrase inhibition ameliorates lipid disorder via suppression of PGC-1a PARylation in db/db mice. Int J Obes. 2020:44(8):1691-702. Available from:. https://doi.org/10.1038/s41366-020-0573-z.
86. Lakshmi TV, Bale S, Khurana A, Godugu C. Tankyrase as a Novel Molecular Target in Cancer and Fibrotic Diseases. Curr Drug Targets. 2016;18(10):121424

87. Deng Z, Lezina L, Chen CJ, Shtivelband S, So W, Lieberman PM. Telomeric proteins regulate episomal maintenance of epstein-barr virus origin of plasmid replication. Mol Cell. 2002;9(3):493-503. Available from:. https://doi. org/10.1016/S1097-2765(02)00476-8.

88. Li Z, Yamauchi Y, Kamakura M, Murayama T, Goshima F, Kimura H, et al. Herpes simplex virus requires poly (ADP-ribose) polymerase activity for efficient replication and induces extracellular signal-related kinasedependent phosphorylation and ICP0-dependent nuclear localization of Tankyrase 1. J Virol. 2012;86(1):492-503. https://doi.org/10.1128/JVI.05897-11.

89. McGurk L, Rifai O, Bonini NM. TDP-43 a protein central to amyotrophic lateral sclerosis is destabilized by Tankyrase-1/2. J Cell Sci. 2020;133(12): jcs245811.

90. Cerami E, Gao J, Dogrusoz U, Gross BE, Sumer SO, Aksoy BA, et al. The cBio Cancer genomics portal: an open platform for exploring multidimensional cancer genomics data. Cancer Discov. 2012;2(5):401-4. https://doi.org/10.11 58/2159-8290.CD-12-0095.

91. Gao J, Aksoy BA, Dogrusoz U, Dresdner G, Gross B, Sumer SO, et al. Integrative analysis of complex cancer genomics and clinical profiles using the cBioPortal. Sci Signal. 2013;6(269):pl1.

92. Schatoff EM, Goswami S, Zafra MP, Foronda M, Shusterman M, Leach Bl, et al. Distinct colorectal cancer-associated apc mutations dictate response to tankyrase inhibition. Cancer Discov. 2019;9(10):1358-71. https://doi.org/1 0.1158/2159-8290.CD-19-0289.

93. Jang MK, Mashima T, Seimiya H. Tankyrase inhibitors target colorectal cancer stem cells via axin-dependent downregulation of c-kit tyrosine kinase. Mol Cancer Ther. 2020;19(3):765-76. https://doi.org/10.1158/1535-71 63.MCT-19-0668.

94. Busch AM, Johnson KC, Stan RV, Sanglikar A, Ahmed Y, Dmitrovsky E, et al. Evidence for tankyrases as antineoplastic targets in lung cancer. BMC Cancer. 2013;13:1-15.

95. Tang B, Wang J, Fang J, Jiang B, Zhang M, Wang $Y$, et al. Expression of TNKS1 is correlated with pathologic grade and Wnt/ $\beta$-catenin pathway in human astrocytomas. J Clin Neurosci. 2012;19(1):139-43. Available from:. https://doi.org/10.1016/j.jocn.2011.08.013.

96. Bao R, Christova T, Song S, Angers S, Yan X, Attisano L. Inhibition of Tankyrases Induces Axin Stabilization and Blocks Wnt Signalling in Breast Cancer Cells. PLoS One. 2012;7(11):e48670.

97. Yang HY, Shen JX, Wang Y, Liu Y, Shen DY, Quan S. Tankyrase promotes aerobic glycolysis and proliferation of ovarian Cancer through activation of Wnt/ $\beta$-catenin signaling. Biomed Res Int. 2019;2019:2686340.

98. Ma L, Wang X, Jia T, Wei W, Chua MS, So S. Tankyrase inhibitors attenuate WNT/ß-catenin signaling and inhibit growth of hepatocellular carcinoma cells. Oncotarget. 2015;6(28):25390-401. https://doi.org/10.18632/oncotarget.4455.

99. Huang SMA, Mishina YM, Liu S, Cheung A, Stegmeier F, Michaud GA, et al, Tankyrase inhibition stabilizes axin and antagonizes Wnt signalling. Nature. 2009;461(7264):614-20. Available from:. https://doi.org/10.1038/nature08356.

100. Norum JH, Skarpen E, Brech A, Kuiper R, Waaler J, Krauss S, et al. The tankyrase inhibitor G007-LK inhibits small intestine LGR5+ stem cell proliferation without altering tissue morphology. Biol Res. 2018;51(1):1-13.

101. Mizutani A, Yashiroda Y, Muramatsu Y, Yoshida H, Chikada T, Tsumura T, et al. RK-287107, a potent and specific tankyrase inhibitor, blocks colorectal cancer cell growth in a preclinical model. Cancer Sci. 2018;109(12):4003-14. https://doi.org/10.1111/cas.13805.

102. Okada-Iwasaki R, Takahashi Y, Watanabe Y, Ishida H, Saito Jl, Nakai R, et al. The discovery and characterization of K-756, a novel Wnt/b-catenin pathway inhibitor targeting tankyrase. Mol Cancer Ther. 2016;15(7):1525-34. https://doi.org/10.1158/1535-7163.MCT-15-0938.

103. Nusse $R$, Clevers H. Wnt/ $\beta$-Catenin Signaling, Disease, and Emerging Therapeutic Modalities. Cell. 2017;169:985-99 Cell Press.

104. Ha GH, Kim DY, Breuer EK, Kim CK. Combination treatment of polo-like kinase 1 and tankyrase-1 inhibitors enhances anticancer effect in triplenegative breast cancer cells. Anticancer Res. 2018;38(3):1303-10.

105. Tian X, Hou W, Bai S, Fan J, Tong H, Bai Y. XAV939 promotes apoptosis in a neuroblastoma cell line via telomere shortening. Oncol Rep. 2014;32(5): 1999-2006. https://doi.org/10.3892/or.2014.3460.

106. Jia J, Qiao Y, Pilo MG, Cigliano A, Liu X, Shao Z, et al. Tankyrase inhibitors suppress hepatocellular carcinoma cell growth via modulating the hippo cascade. PLoS One. 2017;12(9):1-15. 
107. Thorvaldsen TE, Pedersen NM, Wenzel EM, Schultz SW, Brech A, Liestøl K, et al. Structure, dynamics, and functionality of tankyrase inhibitor-induced degradasomes. Mol Cancer Res. 2015;13(11):1487-501. https://doi.org/10.11 58/1541-7786.MCR-15-0125.

108. Kierulf-Vieira KS, Sandberg CJ, Waaler J, Lund K, Skaga E, Saberniak BM, et al. A small-molecule tankyrase inhibitor reduces glioma stem cell proliferation and sphere formation. Cancers (Basel). 2020;12(6):1-16.

109. Waaler J, Mygland L, Tveita A, Strand MF, Solberg NT, Olsen PA, et al. Tankyrase inhibition sensitizes melanoma to PD-1 immune checkpoint blockade in syngeneic mouse models. Commun Biol [Internet]. 2020;3(1):113 Available from: https://doi.org/10.1038/s42003-020-0916-2.

110. Anumala UR, Waaler J, Nkizinkiko $Y$, Ignatev A, Lazarow $K$, Lindemann $P$, et al. Discovery of a novel series of Tankyrase inhibitors by a hybridization approach. J Med Chem. 2017;60(24):10013-25. https://doi.org/10.1021/acs. jmedchem.7b00883.

111. Waaler J, Leenders RGG, Sowa ST, Alam Brinch S, Lycke M, Nieczypor $P$, et al. Preclinical Lead optimization of a 1,2,4-Triazole based Tankyrase inhibitor. J Med Chem. 2020;63(13):6834-46. https://doi.org/10.1021/acs.jmedchem. 0c00208.

112. Scarborough HA, Helfrich BA, Casas-Selves M, Schuller AG, Grosskurth SE, Kim J, et al. AZ1366: an inhibitor of tankyrase and the canonical wnt pathway that limits the persistence of non-small cell lung cancer cells following EGFR inhibition. Clin Cancer Res. 2017;23(6):1531-41. https://doi. org/10.1158/1078-0432.CCR-16-1179.

113. Wang $H$, Castillo J, Zhang Y, Yang Z, McAllister G, Lindeman A, et al. Tankyrase inhibitor sensitizes lung cancer cells to endothelial growth factor receptor (EGFR) inhibition via stabilizing angiomotins and inhibiting yap signaling. J Biol Chem. 2016;291(29):15256-66. https://doi.org/10.1074/jbc. M116.722967.

114. Huang J, Qu Q, Guo Y, Xiang Y, Feng D. Tankyrases/ß-catenin signaling pathway as an anti-proliferation and anti-metastatic target in hepatocarcinoma cell lines. J Cancer. 2020;11(2):432-40. https://doi.org/10. 7150/jca.30976.

115. Shirai F, Mizutani A, Yashiroda Y, Tsumura T, Kano Y, Muramatsu Y, et al. Design and discovery of an orally efficacious Spiroindolinone-based Tankyrase inhibitor for the treatment of Colon Cancer. J Med Chem. 2020; 63(8):4183-204. https://doi.org/10.1021/acs.jmedchem.0c00045.

116. Li B, Liang J, Lu F, Zeng G, Zhang J, Ma Y, et al. Discovery of novel inhibitor for Wnt/ $\beta$-catenin pathway by tankyrase 1/2 structure-based virtual screening. Molecules. 2020;25(7):1680.

117. Song M, Emilsson L, Bozorg SR, Nguyen LH, Joshi AD, Staller K, et al. Risk of colorectal cancer incidence and mortality after polypectomy: a Swedish record-linkage study. Lancet Gastroenterol Hepatol. 2020 Jun;5(6):537-47. https://doi.org/10.1016/S2468-1253(20)30009-1.

118. Polakis P. Drugging Wnt signalling in cancer. EMBO J. 2012;31:2737-46 Wiley-VCH Verlag.

119. Voronkov A, Holsworth DD, Waaler J, Wilson SR, Ekblad B, Perdreau-Dahl H, et al. Structural basis and SAR for G007-LK, a lead stage 1,2,4-triazole based specific tankyrase 1/2 inhibitor. J Med Chem. 2013;56(7):3012-23. https://doi. org/10.1021/jm4000566.

120. Lau T, Chan E, Callow M, Waaler J, Boggs J, Blake RA, et al. A novel tankyrase small-molecule inhibitor suppresses APC mutation-driven colorectal tumor growth. Cancer Res. 2013;73(10):3132-44. https://doi.org/1 0.1158/0008-5472.CAN-12-4562

121. Tanaka N, Mashima T, Mizutani A, Sato A, Aoyama A, Gong B, et al. APC mutations as a potential biomarker for sensitivity to tankyrase inhibitors in colorectal cancer. Mol Cancer Ther. 2017;16(4):752-62. https://doi.org/10.11 58/1535-7163.MCT-16-0578.

122. Solberg NT, Waaler J, Lund K, Mygland L, Olsen PA, Krauss S. TANKYRASE inhibition enhances the antiproliferative effect of PI3K and EGFR inhibition, mutually affecting $\beta$-CATENIN and AKT signaling in colorectal cancer. Mol Cancer Res. 2018;16(3):543-53. https://doi.org/10.1158/1541-7786.MCR-17-03 62.

123. Li C, Zheng X, Han Y, Lv Y, Lan F, Zhao J. XAV939 inhibits the proliferation and migration of lung adenocarcinoma A549 cells through the WNT pathway. Oncol Lett. 2018;15(6):8973-82. https://doi.org/10.3892/ol.2018.84 91.

124. Uematsu K, He B, You L, Xu Z, McCormick F, Jablons DM. Activation of the Wnt pathway in non small cell lung cancer: evidence of dishevelled overexpression. Oncogene. 2003;22(46):7218-21. https://doi.org/10.1038/sj. onc.1206817.
125. Kulak O, Chen H, Holohan B, Wu X, He H, Borek D, et al. Disruption of Wnt/ $\beta$-catenin signaling and Telomeric shortening are inextricable consequences of Tankyrase inhibition in human cells. Mol Cell Biol. 2015;35(14):2425-35.

126. Shultz MD, Cheung AK, Kirby CA, Firestone B, Fan J, Chen CHT, et al. Identification of NVP-TNKS656: the use of structure-efficiency relationships to generate a highly potent, selective, and orally active tankyrase inhibitor. J Med Chem. 2013;56(16):6495-511. https://doi.org/10.1021/jm400807n.

127. Casás-Selves M, Kim J, Zhang Z, Helfrich BA, Gao D, Porter CC, et al. Tankyrase and the canonical Wnt pathway protect lung cancer cells from EGFR inhibition. Cancer Res. 2012;72(16):4154-64. https://doi.org/10.1158/ 0008-5472.CAN-11-2848

128. Xu W, Lau YH, Fischer G, Tan YS, Chattopadhyay A, De La Roche M, et al. Macrocyclized extended peptides: inhibiting the substrate-recognition domain of Tankyrase. J Am Chem Soc. 2017;139(6):2245-56. https://doi. org/10.1021/jacs.6b10234.

129. Xu D, Liu J, Fu T, Shan B, Qian L, Pan L, et al. USP25 regulates WNT signalling by controlling the stability of tankyrases. Genes Dev. 2017;31(10): 1024-35. https://doi.org/10.1101/gad.300889.117.

130. Cheng H, Li X, Wang C, Chen Y, Li S, Tan J, et al. Inhibition of tankyrase by a novel small molecule significantly attenuates prostate cancer cell proliferation. Cancer Lett. 2019;443(November 2018):80-90. https://doi.org/1 0.1016/j.canlet.2018.11.013

131. Pollock K, Liu M, Zaleska M, Meniconi M, Pfuhl M, Collins I, et al. Fragmentbased screening identifies molecules targeting the substrate-binding ankyrin repeat domains of tankyrase. Sci Rep. 2019;9(1):1-20.

132. Sowa ST, Vela-Rodríguez C, Galera-Prat A, Cázares-Olivera M, PrunskaiteHyyryläinen R, Ignatev $A$, et al. A FRET-based high-throughput screening platform for the discovery of chemical probes targeting the scaffolding functions of human tankyrases. Sci Rep. 2020;10(1):1-12. Available from. https://doi.org/10.1038/s41598-020-69229-y.

\section{Publisher's Note}

Springer Nature remains neutral with regard to jurisdictional claims in published maps and institutional affiliations.

Ready to submit your research? Choose BMC and benefit from:

- fast, convenient online submission

- thorough peer review by experienced researchers in your field

- rapid publication on acceptance

- support for research data, including large and complex data types

- gold Open Access which fosters wider collaboration and increased citations

- maximum visibility for your research: over $100 \mathrm{M}$ website views per year

At BMC, research is always in progress.

Learn more biomedcentral.com/submissions 